\title{
Tuberculosis congénita asociada con tuberculosis materna miliar diseminada
}

\author{
Luis Miguel Sosa, Luz Libia Cala, Julio César Mantilla \\ Departamento de Pediatría, Universidad Industrial de Santander, Hospital Universitario de Santander, \\ Bucaramanga, Colombia
}

\begin{abstract}
La tuberculosis sin tratamiento en mujeres gestantes es un riesgo definido para la transmisión de la enfermedad al recién nacido y para resultados adversos, obstétricos y perinatales. La tuberculosis en mujeres gestantes y la tuberculosis congénita son afecciones infrecuentes y de difícil diagnóstico por la falta de especificidad de las manifestaciones clínicas.

Se presenta el caso de una mujer primigestante con tuberculosis miliar diseminada, con inicio de las manifestaciones en el puerperio inmediato, diagnóstico en el segundo mes del puerperio y desenlace fatal. Además, se presenta el caso de su hijo prematuro con manifestaciones desde el nacimiento, evidencia de complejo pulmonar primario, atelectasia persistente por obstrucción bronquial por las adenopatías e infección por citomegalovirus; recibió tratamiento estándar con mejoría.
\end{abstract}

Palabras clave: tuberculosis/congénito, tuberculosis miliar, tuberculosis ganglionar, embarazo, síndrome de dificultad respiratoria del recién nacido, sepsis, citomegalovirus.

\section{Congenital tuberculosis associated with maternal disseminated miliary tuberculosis}

Untreated tuberculosis during pregnancy presents a serious risk for transmission of disease to the newborn and can result in adverse perinatal and obstetrical outcomes. Tuberculosis during pregnancy and congenital tuberculosis are infrequent conditions and are difficult to diagnose due the non-specificity of the symptoms.

A case report is presented of a woman who had no children previously with disseminated miliary tuberculosis. Tuberculosis symptoms appeared immediately after birth of the first child, with a clinical diagnosis on the second month after childbirth, whereupon the patient died. The son, a premature infant, showed disease symptoms from the first day, with primary pulmonary complex and persistent atelectasis due to bronchial obstruction. The obstruction was due to thoracic lymphadenitis and coinfection with cytomegalovirus. The infant received standard treatment and his condition improved.

Key words: tuberculosis/congenital; tuberculosis, miliary; tuberculosis, lymph node; pregnancy; respiratory distress syndrome, newborn; sepsis; cytomegalovirus.

La tuberculosis ha permanecido a lo largo de la historia como un grave problema de salud pública que afecta todas las edades, a pesar de los avances científicos y de un tratamiento efectivo (1). La incidencia de tuberculosis en mujeres

\footnotetext{
Correspondencia:

Luis Miguel Sosa, Departamento de Pediatría, Hospital Universitario de Santander, cuarto piso, Bucaramanga, Colombia.

Teléfono: 6419664

lumisosa@gmail.com

Recibido: 30/03/07; aceptado:13/09/07
}

gestantes y en edad fértil está directamente relacionada con su prevalencia en la población general y con la prevalencia de infección por el virus de la inmunodeficiencia humana $(\mathrm{VIH})(2,3)$. El $53 \%$ de los casos de tuberculosis reportados en Colombia en el 2005 corresponde a personas entre los 15 y los 44 años (4).

La tuberculosis sin tratamiento en mujeres gestantes es un riesgo definido de transmisión de la enfermedad al recién nacido; de allí que se requiera una atención especial en este tipo de 
pacientes (3). El aumento del número de casos entre mujeres gestantes hace suponer un incremento de la aparición de tuberculosis congénita, la cual es una entidad rara, de difícil diagnóstico por la falta de especificidad de las manifestaciones clínicas, de curso rápido y progresivo y con una alta mortalidad (5-7).

En el presente artículo, se presenta un caso de tuberculosis miliar diseminada en una mujer gestante que falleció durante el puerperio, y el de su hijo con tuberculosis congénita sin desenlace fatal, al igual que las dificultades en el diagnóstico y en el tratamiento debido a la infección asociada con citomegalovirus (CMV).

\section{Historia clínica de la madre}

Se presenta el caso de una mujer de 20 años de edad, primigestante, con un embarazo de 30 semanas y tres controles prenatales sin complicaciones. Presentó trabajo prematuro de parto, fiebre y taquicardia fetal. El producto nació por parto vaginal, sin complicaciones. La paciente no amamantó a su hijo por la enfermedad del recién nacido.

A partir del décimo día de puerperio presentó fiebre, tos seca, síntomas constitucionales, como astenia, adinamia e hiporexia, náuseas y vómito, cefalea global, alteraciones de la conducta y alteración del estado de conciencia con desorientación témporo-espacial.

En el segundo mes del puerperio se le practicó punción lumbar y se obtuvo un líquido cefalorraquídeo con las siguientes características: 18 leucocitos $/ \mathrm{mm}^{3}$, con $36 \%$ de segmentados, y un índice glucorraquia (48 mg/dl)/glucemia (106 mg/dl) de 0,45 , con $45 \mathrm{mg} / \mathrm{dl}$ de proteínas. Se inició manejo con ceftriaxona y vancomicina.

Ante la falta de respuesta al tratamiento, se tomó una radiografía de tórax en la que se observaba un infiltrado pulmonar intersticial, tipo micronodular, difuso y bilateral. Se inició tratamiento antituberculoso. Presentó deterioro neurológico progresivo y en la tomografía axial computadorizada (TAC) cerebral se apreció una lesión isquémica fronto-temporal derecha, la cual se consideró como secundaria a vasculitis del sistema nervioso central. La serología para VIH fue negativa en dos exámenes diferentes. Hubo empeoramiento de su cuadro clínico y falleció en el segundo mes después del parto.

En la necropsia se encontró tuberculosis miliar generalizada, con granulomas y necrosis de caseificación, con la coloración de Ziehl Nielsen (ZN) positiva en nuestras de cerebro, útero, ovario, trompas, peritoneo, ganglios del cuello y mediastino, pulmón, hígado, y bazo (figura 1); además, vasculitis del sistema nervioso central, con lesión isquémica en la región témporo-parietal izquierda.

\section{Historia clínica del niño}

Se trata del primogénito de la mujer primigestante de 20 años. Nació por parto vaginal, prematuro (30 semanas de edad de gestación), de sexo masculino, $1.530 \mathrm{~g}$ de peso, $32 \mathrm{~cm}$ de perímetro cefálico, $42 \mathrm{~cm}$ de talla, y con índice de Apgar de 8 al minuto y de 9 a los 5 minutos.

Presentó dificultad respiratoria en las primeras horas de vida, que requirió respiración asistida. Durante la hospitalización, en las primeras tres semanas de vida, presentó síndrome de respuesta inflamatoria sistémica con trombocitopenia y hemocultivos negativos, y conjuntivitis por Enterobacter sp., por lo que recibió terapia antibiótica. Presentó mejoría y fue dado de alta.

Consultó nuevamente a los dos meses de edad por enfermedad diarreica aguda, tos y palidez. En el examen físico se encontró: peso de $2,9 \mathrm{~kg}$, perímetro cefálico de $36,5 \mathrm{~cm}$, frecuencia respiratoria de 60 por minuto, frecuencia cardiaca de 166 pulsaciones por minuto, saturación de oxígeno de $87 \%$ con aire ambiente, hipoactivo, palidez generalizada, tirajes subcostales e intercostales y retracción subxifoidea leve. En la radiografía de tórax se apreciaban infiltrados intersticiales bilaterales y atelectasia retrocardiaca izquierda (figura 2). En el examen de sangre se encontró hemoglobina de $6,8 \mathrm{~g} \%$, hematocrito de $21 \%$ y 14.700 leucocitos. Se inició tratamiento con claritromicina por sospecha de neumonía atípica y se hizo transfusión de glóbulos rojos.

En las dos primeras semanas de hospitalización hubo empeoramiento de la dificultad respiratoria, obstrucción bronquial, cianosis y desaturación de oxígeno, hepatoesplenomegalia y síndrome de 

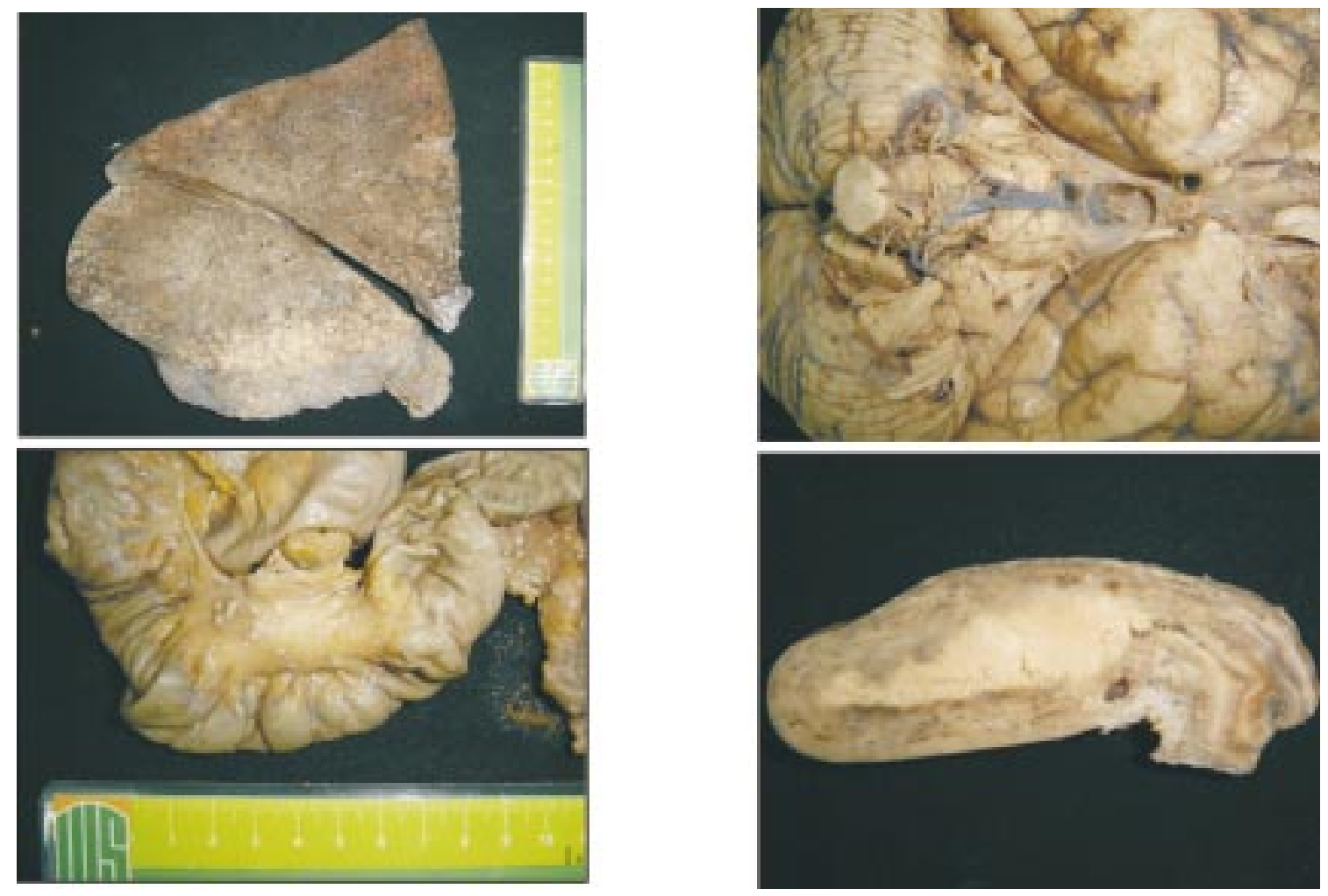

Figura 1. Hallazgos macroscópicos de la necropsia de la madre. Se aprecian abundantes granulomas en pulmón, intestino, base del encéfalo con exudado fibrinoso y útero con gran granuloma en su interior.
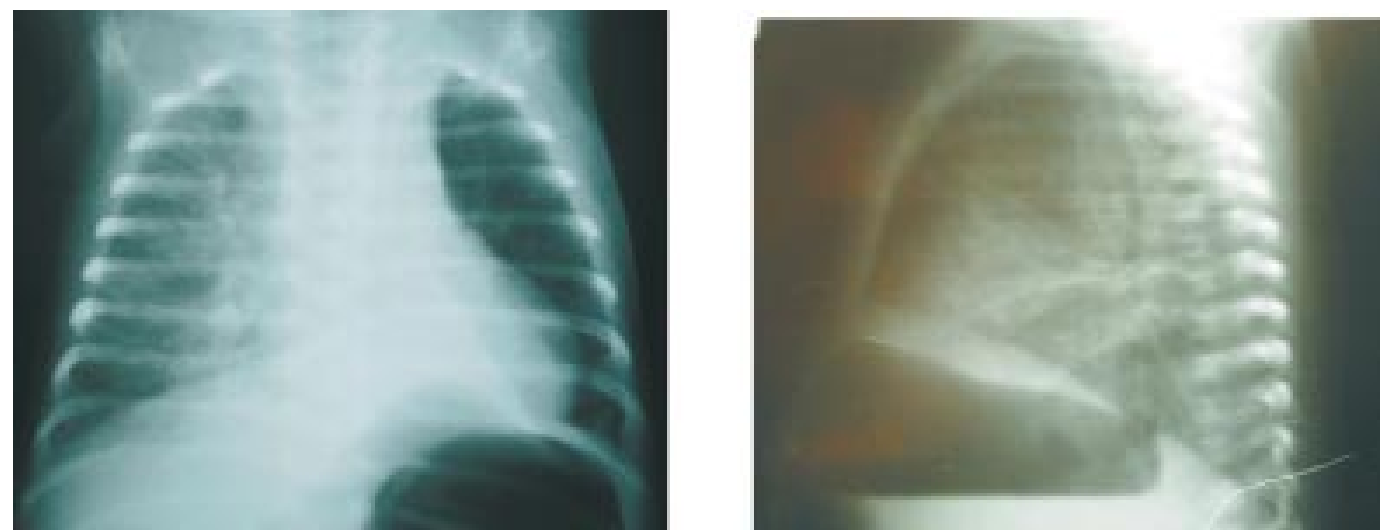

Figura 2. Radiografía de tórax antero-posterior y lateral. Se aprecian infiltrados intersticiales bilaterales y atelectasia retrocardiaca izquierda.

respuesta inflamatoria sistémica. En la radiografía de tórax se observaba consolidación neumónica en la base derecha. Los hemocultivos fueron negativos, hubo trombocitopenia y la LDH (lactodeshidrogenasa) fue de $621 \mathrm{UI} / \mathrm{l}$. Se inició terapia antibiótica con cefotaxime.
Se obtuvo la información de que la madre se encontraba hospitalizada con meningitis tuberculosa. Se practicaron cuatro baciloscopias de jugo gástrico, dos de las cuales resultaron positivas; la serología para el VIH fue negativa. Se inició manejo con isoniacida, rifampicina y 
pirazinamida. Se le tomó TAC de tórax, que se aprecia en la figura 3.

Durante la tercera y la cuarta semanas de hospitalización, presentó tos metálica y persistían la dificultad respiratoria y el síndrome broncoobstructivo. En la TAC de tórax se apreciaban ganglios supraaórticos y pretraqueales, disminución del calibre de la carina, colapso del bronquio fuente izquierdo, consolidación, necrosis y atelectasia del lóbulo inferior izquierdo y callo óseo en el séptimo arco costal posterior izquierdo.
La TAC abdominal fue normal. La IgM anti-CMV fue positiva. La valoración por oftalmología no detectó anormalidades. Se decidió iniciar corticoides por la tuberculosis ganglionar y ganciclovir por el riesgo de progresión del CMV, aunque no hubo certeza de una infección asociada.

Durante el segundo mes de hospitalización presentó sepsis por Acinetobactersp. y Klebsiella sp., y recibió tratamiento antibiótico con meropenem. Hubo mejoría gradual de la dificultad respiratoria.
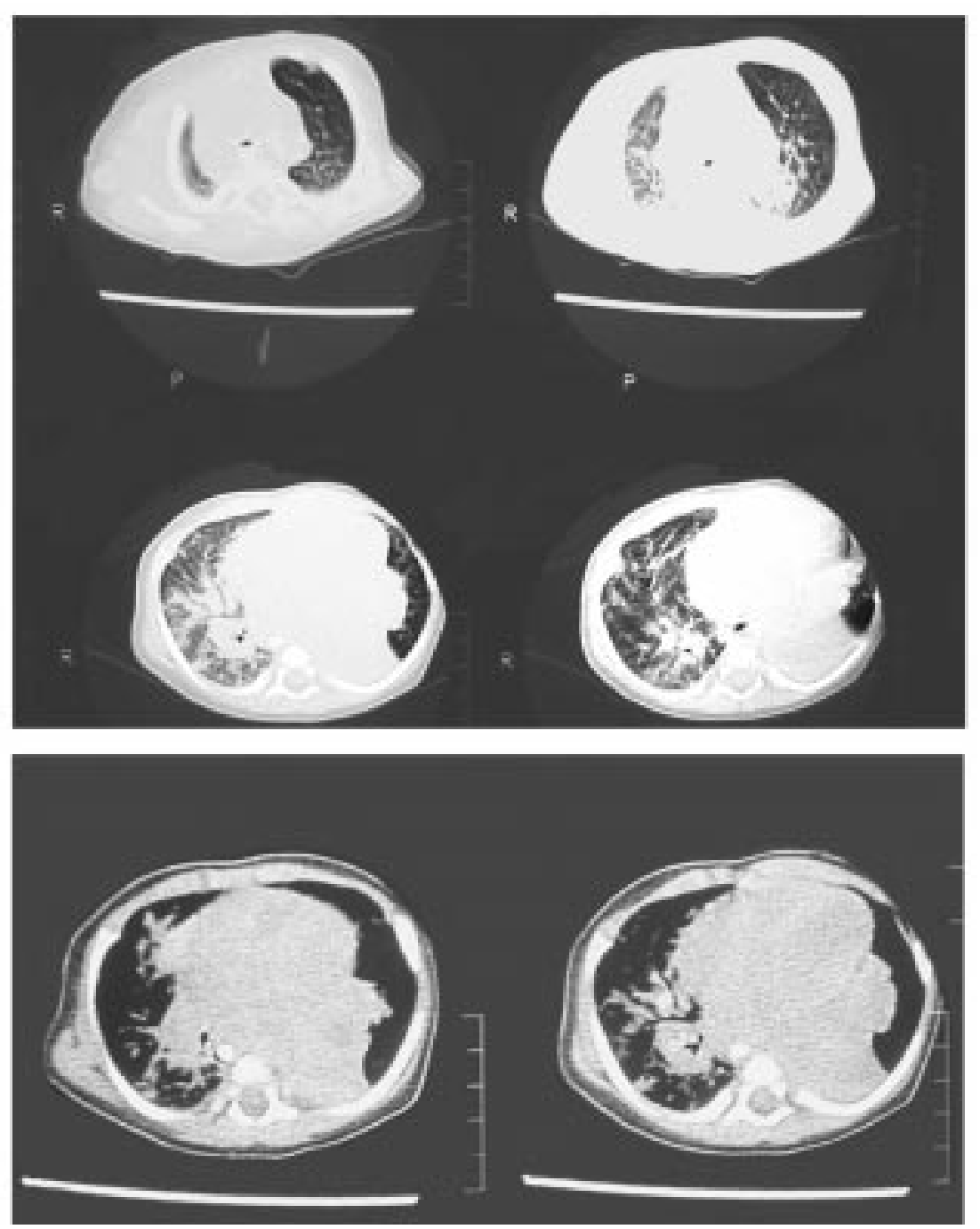

Figura 3. TAC de tórax con ventana para pulmón, a los dos meses y medio de edad, en el momento del diagnóstico de tuberculosis. Se aprecian atelectasia del lóbulo inferior izquierdo, adenopatías mediastinales y consolidación del lóbulo inferior derecho y del lóbulo medio. 


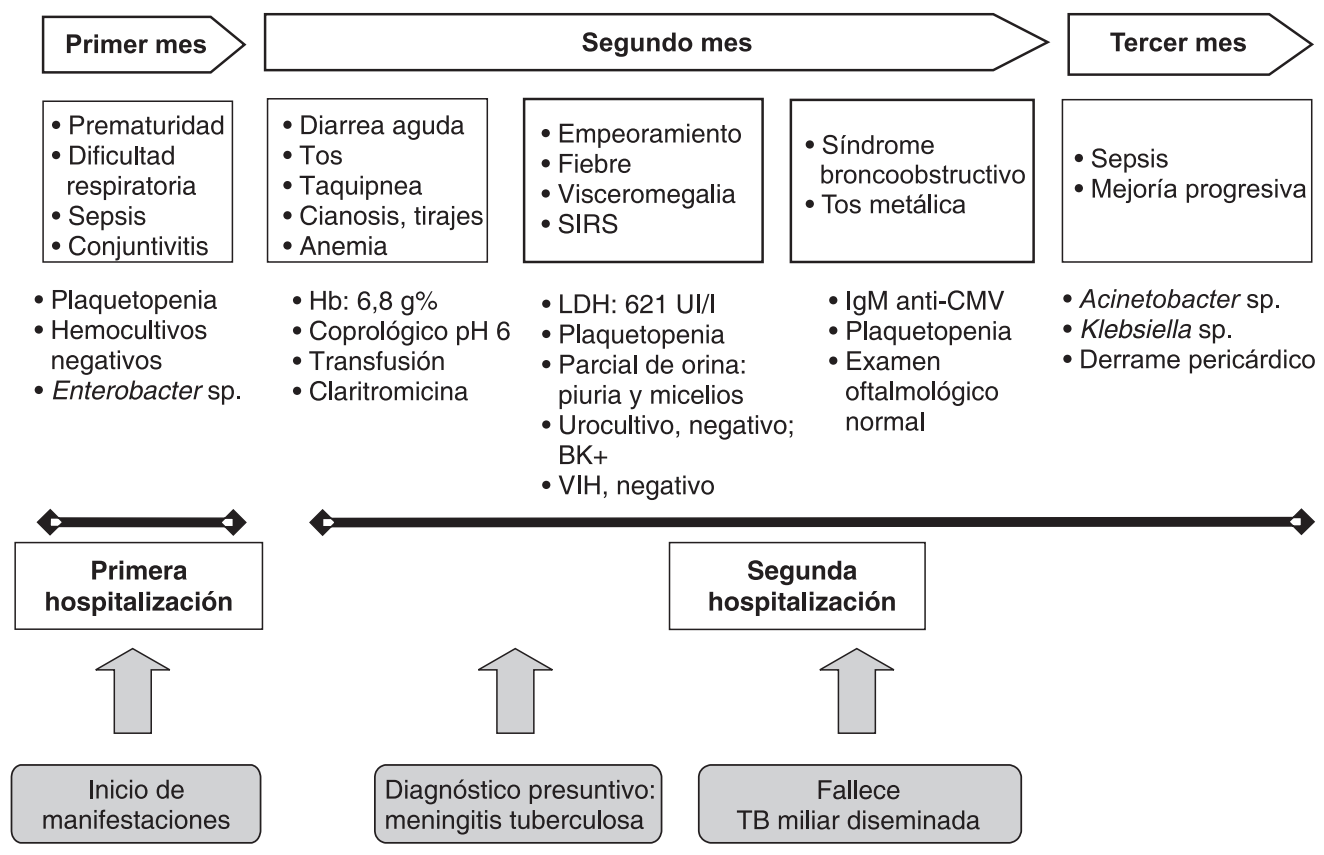

Figura 4. Curso clínico del niño. Las flechas superiores sin relleno corresponden a la edad cronológica del niño. Las figuras geométricas sin relleno corresponden al curso clínico del niño y las figuras en sombreado gris corresponden a los acontecimientos clínicos de la madre. Las líneas gruesas indican la duración de cada hospitalización.

Se le dio de alta por mejoría del cuadro clínico. A los cinco meses de edad fue hospitalizado nuevamente con síndrome broncoobstructivo moderado y a los ocho meses por neumonía. Actualmente, terminó la segunda fase de tratamiento acortado supervisado y está en seguimiento ambulatorio (figura 4).

\section{Discusión}

La tuberculosis impone una carga importante al mundo y constituye la primera causa de muerte por infecciones entre las mujeres (8), $80 \%$ de las cuales ocurren en la edad reproductiva (9). En Colombia, la letalidad para el 2006 fue de $0,3 \%$ (10), $16 \%$ de los casos reportados en el 2005 fueron de tuberculosis extrapulmonar y más de la mitad ocurrieron en personas en edad fértil (4). El caso de la paciente gestante del presente informe corresponde a una mujer joven con tuberculosis miliar diseminada que falleció durante el puerperio.

Desde la época de Hipócrates y hasta el siglo XIV, se creía que el embarazo mejoraba el curso de la tuberculosis (2). Luego, se demostró que la tuberculosis empeoraba en el embarazo (2). La progresión es similar en mujeres gestantes y no gestantes (11); durante el embarazo, 84\% permanecía sin cambios, 9\% mejoraba y $7 \%$ empeoraba; y, durante el puerperio, $76 \%$ permanecía sin cambios, $9 \%$ mejoraba y $15 \%$ empeoraba $(2,9)$. Se desconoce el efecto que pudo haber tenido el embarazo sobre la progresión de la tuberculosis en nuestra paciente.

La tuberculosis pulmonar activa en la mujer gestante no se ha asociado con malformaciones congénitas pero sí con un riesgo mayor de parto prematuro, retardo del crecimiento intrauterino, bajo peso al nacer y mortalidad perinatal $(2,11,12)$. La tuberculosis extrapulmonar diferente a la ganglionar se asocia con Apgar bajo y bajo peso al nacer (12). En nuestro caso, el recién nacido presentó parto prematuro y bajo peso al nacer.

La mujer gestante con tuberculosis pulmonar puede presentar tos (47\%), pérdida de peso (41\%) y síntomas constitucionales, como fiebre malestar y fatiga (30\%), aunque $20 \%$ pueden ser asintomáticas y tener estudios radiológicos anormales (12-14). Las manifestaciones de 
tuberculosis extrapulmonar, más frecuentes en los países desarrollados, dependen de la localización $(10,12,14)$. En nuestro caso, la madre presentó síntomas sugestivos de corioamnionitis durante el trabajo de parto prematuro que podría explicarse por la presencia de granulomas en la cavidad uterina. Los síntomas de afección del sistema nervioso central aparecieron en el décimo día del puerperio; se sospechó meningitis tuberculosa en el segundo mes del puerperio y el diagnóstico definitivo se obtuvo post mórtem.

El retardo en el diagnóstico de tuberculosis en la gestación puede obedecer a la presentación tardía de manifestaciones, a la falta de especificidad de los síntomas, al cumplimiento deficiente a las citas de control prenatal o a la necesidad de diferir los estudios radiográficos $(10,14)$.

Los síntomas como astenia, adinamia, letargia y las alteraciones en el hábito intestinal pueden estar presentes en la mujer gestante sin tuberculosis; de igual forma, las manifestaciones extrapulmonares son inespecíficas y de aparición tardía $(10,14)$. Dieciséis por ciento de las mujeres gestantes con tuberculosis se diagnosticaron en el primer trimestre de gestación, $31 \%$ en el segundo trimestre, $3 \%$ en el tercer trimestre y $22 \%$ después del parto (14). En $50 \%$ de los casos, el diagnóstico de la madre se estableció a partir del diagnóstico en el niño (5). En nuestro caso, el diagnóstico del niño se realizó a partir del diagnóstico de la madre en el segundo mes del puerperio.

Para el diagnóstico de tuberculosis durante la gestación se necesita un alto grado de sospecha que requiere el conocimiento de la historia previa de tuberculosis o de contactos, la identificación de síntomas sugestivos de la enfermedad y la realización de pruebas, como la tuberculina, obtención y lectura de una baciloscopia y radiografía de tórax $(2,13)$. Ante una prueba de tuberculina positiva con radiografía de tórax normal, se debería iniciar quimioprofilaxis con isoniacida durante la gestación o después del parto, dependiendo de la probabilidad de haber sido recientemente infectada y del riesgo de progresión de la enfermedad $(2,13,15)$. Si se presenta una prueba positiva de tuberculina y una radiografía anormal de tórax o evidencia de enfermedad o del bacilo, se debe iniciar tratamiento con medicamentos no teratogénicos, como la isoniacida, la rifampicina o el etambutol $(2,13,15)$.

La tuberculosis congénita es una afección rara (5-7). En la literatura inglesa se han reportado un poco más de 300 casos. En un estudio se encontraron tres niños afectados entre 100 madres con tuberculosis activa, en tanto que en otras dos series no se encontraron niños afectados, entre 260 y 1.369 madres con tuberculosis activa (5). En América, durante el último siglo, se han reportado casos en México (16), Venezuela (17), Perú (18), Costa Rica (19) y Colombia $(20,21)$. Este es el primer caso reportado en el nororiente colombiano.

Las manifestaciones pueden evidenciarse desde el nacimiento, aunque son más frecuentes entre la segunda y la cuarta semanas de vida $(22,23)$. En una sistematización de 300 reportes, la mediana de la edad de aparición de las manifestaciones fue de 24 días, con un rango de 1 a 84 días (5). En nuestro caso, hubo manifestaciones iniciales en el período neonatal y, posteriormente, manifestaciones respiratorias a las ocho semanas de vida.

La bacteriemia tuberculosa que ocurre durante el embarazo puede resultar en infección de la placenta o del aparato genital materno (24). Esta infección puede ser transmitida al feto por dos mecanismos: 1) a partir de la ruptura de un tubérculo de la placenta ocurre diseminación hematógena por vía del cordón umbilical, que ocasiona un complejo primario en el hígado o en el pulmón $(25,26)$; 2$)$ a partir de una afección de los genitales de la madre (figura 1) ocurre aspiración o deglución de líquido amniótico o sangre infectados, que ocasiona la formación del complejo primario en el pulmón con compromiso de los ganglios mediastinales, o en el intestino con compromiso de los ganglios mesentéricos $(25,26)$. En nuestro caso, el complejo primario se localizó en el pulmón, con compromiso de los ganglios mediastinales, lo que podría sugerir diseminación hematógena o aspiración de líquido amniótico infectado. 
El diagnóstico de tuberculosis congénita es difícil y requiere un alto grado de sospecha, debido a la falta de especificidad de las manifestaciones, a que usualmente hay compromiso multisistémico y a que en la mayoría de los casos se desconoce el estado infeccioso de la madre $(5,22,27)$. La presentación clínica puede semejarse a cualquier otra infección congénita o a sepsis bacteriana. Las manifestaciones más frecuentes incluyen, entre otras: hepatoesplenomegalia, $76 \%$; dificultad respiratoria, $72 \%$; fiebre, $48 \%$; adenopatías, 38\%; distensión abdominal, 24\%, y letargia e irritabilidad, $21 \%$ (5). Es necesario descartar otras causas de infección congénita y complementar el diagnóstico para determinar el compromiso multisistémico, incluido el examen de fondo de ojo $(6,7)$. El caso reportado presentó sepsis neonatal, anemia, trombocitopenia, hepatoesplenomegalia, neumonitis, fiebre y adenopatías mediastinales, sin compromiso ocular; la lgM fue positiva para citomegalovirus, por lo que es imposible determinar a cuál de las dos entidades se debía cada una de las manifestaciones.

La prueba de tuberculina es de utilidad limitada debido a la inmadurez inmunológica, que puede ser más pronunciada en los prematuros (26). La radiografía de tórax puede ser normal al nacimiento y, posteriormente, mostrar una variedad de anormalidades. En $80 \%$ de los pacientes se presentan anormalidades radiográficas como patrón miliar $(50 \%)$, adenopatías, infiltrados parenquimatosos persistentes que, ocasionalmente, llevan a cavitación, e infiltrados inespecíficos $(62 \%)(5,7,28)$. Las lesiones persistentes, como las atelectasias, deben hacer sospechar la enfermedad (21). También, es recomendable complementar los estudios con una TAC de alta resolución $(20,21)$. En nuestro caso, se documentó una variedad de patrones radiológicos: infiltrado intersticial bilateral, condensación y atelectasias persistentes; en la TAC se estableció la presencia de las adenopatías mediastinales y su efecto sobre las estructuras adyacentes.

Beitzki en 1935 (29), Corner en 1952 (30) y Cantwell en 1994 (5) propusieron criterios diagnósticos. Para la confirmación de la tuberculosis congénita proponemos los siguientes criterios: 1) demostración de un foco infeccioso en el aparato genital materno o en la placenta, 2) demostración de Mycobacterium tuberculosis en cualquiera de los líquidos corporales del niño, y 3) presencia del complejo primario en pulmón, intestino o hígado. Para la demostración de $M$. tuberculosis, el examen más sencillo y barato sigue siendo la identificación del bacilo en el aspirado gástrico, mediante tinción de Zielh-Nielsen y cultivo (31), aunque también se pueden obtener aislamientos en otros líquidos corporales, como aspirado traqueal, otorrea, líquido cefalorraquídeo, orina o líquido peritoneal (5). En nuestro caso, el complejo primario estaba en el pulmón y había compromiso de los ganglios mediastinales; la demostración bacteriológica se hizo mediante aspirado de jugo gástrico y se demostró la presencia de granulomas endometriales.

El complejo primario se forma, aproximadamente, a la cuarta semana del contagio y puede evolucionar hacia la curación, extenderse a estructuras contiguas o provocar agrandamiento de los ganglios que provocan un efecto compresivo en las estructuras adyacentes $(22,24)$. En nuestro paciente, se evidenció el efecto compresivo de las adenopatías de la carina, que provocó persistencia y empeoramiento de las manifestaciones respiratorias, con atelectasias y síndrome broncoobstructivo.

El pronóstico depende del diagnóstico temprano y del tratamiento oportuno $(1,22,24)$. La mortalidad se sitúa entre $35 \%$ y $50 \%$ (7). Con tratamiento oportuno, la mortalidad es de $22 \%$ (5). El tratamiento con isoniacida, rifampicina y pirazinamida o etambutol ha demostrado ser efectivo en la tuberculosis congénita y producir una rápida remisión de los síntomas $(1,5,22,24)$. Nuestro paciente se manejó de acuerdo con esos estándares, aunque se le adicionó corticoide debido a la tuberculosis ganglionar (1). También, recibió ganciclovir por la posibilidad de progresión de una infección por citomegalovirus.

\section{Conflicto de intereses}

Declaramos no tener conflictos de intereses.

\section{Financiación}

Esta publicación fue financiada por los autores. 


\section{Referencias}

1. American Academy of Pediatrics. Tuberculosis. En: Pickering LK ,editores. Red Book (Libro Rojo). Memoria del Comité de Enfermedades Infecciosas para 2003. 26aㅡ ed. México: Intersistemas; 2004. p.667-88.

2. Khilnani GC. Tuberculosis and pregnancy. Indian J Chest Dis Allied Sci. 2004;46:105-11.

3. World Health Organization. Global tuberculosis control: surveillance, planning, and financing. WHO report 2005 (WHO/HTM/TB/2005.349). Geneva: World Health Organization; 2005.

4. Nova OP. Informe semestral VIH/sida, sexto período epidemiológico julio 2006. Inf Quinc Epidemiol Nac. 2006;11:265-80.

5. Cantwell MF, Shehab ZM, Costello AM, Sands L, Green WF, Ewing EP Jr, et al. Brief report: congenital tuberculosis. N Engl J Med. 1994;330:1051-4.

6. Smith KC. Congenital tuberculosis: a rare manifestation of a common infection. Curr Opin Infect Dis. 2002;15:269-74.

7. Walls T, Shingadia D. Global epidemiology of paediatric tuberculosis. J Infect. 2004;48:13-22.

8. World Health Organization. Global tuberculosis control: surveillance, planning, and financing. WHO report 2007 (WHO/HTM/TB/2007.376). Geneva: World Health Organization; 2007.

9. Tripathy $\mathbf{S N}$, Tripathy $\mathbf{S N}$. Tuberculosis and pregnancy. Int J Gynaecol Obstet. 2003;80:247-53.

10. Llewelyn M, Cropley I, Wilkinson RJ, Davidson RN. Tuberculosis diagnosed during pregnancy: a prospective study from London. Thorax. 2000;55:129-32.

11. Jana N, Vasista K, Jindal SK, Khunnu B, Ghosh K. Perinatal outcome in pregnancies complicated by pulmonary tuberculosis. Int J Gynaecol Obstet. 1994; $44: 119-24$

12. Narayan J, Kala V, Subhas S, Kushagradhi G. Obstetrical outcomes among women with extrapulmonary tuberculosis. N Engl J Med. 1999;341:645-9.

13. Ormerod $\mathbf{P}$. Tuberculosis in pregnancy and the puerperium. Thorax. 2001;56:494-9.

14. Kothari A, Mahadevan N, Girling J. Tuberculosis and pregnancy results of a study in a high prevalence area in London. Eur J Obstet Gynecol Reprod Biol. 2006;126:48-55.

15. Skevaki CL, Kafetzis DA. Tuberculosis in neonates and infants: epidemiology, pathogenesis, clinical manifestations, diagnosis, and management issues. Paediatr Drugs. 2005;7:219-34.

16. Islas LP, Jiménez JR. Tuberculosis pulmonar durante el embarazo: manejo del recién nacido. A propósito de un caso. Revista Mexicana de Pediatría. 2005;72: 298-300.

17. Rodríguez-Manzanero Z, González-Martínez G, Chacón I, García-Martínez V. Tuberculosis y embarazo: reporte de un caso. Kasmera. 2004;32:36-42.

18. Huanca R, Castillo Y, Canales J. Tuberculosis congénita en el Hospital de Quillabamba-Cusco. Rev Situa. 2001;9:57-60.

19. Ivankovich-Escoto $\mathbf{G}$. Tuberculosis congénita: presentación de un caso y revisión de la literatura. Acta Pediátrica Costarricense 2005;19:26-31.

20. Osorio MA, Bolívar G, López P. Tuberculosis congénita. Informe de un caso con autopsia. Colombia Médica. 2000;31:185-8.

21. David M, Ojeda $\mathbf{P}$. Tuberculosis congénita. Presentación de caso clínico. Revista Colombiana de Neumología. 2004;16. [Consultado: 26 de enero de 2007]. Disponible en: http://encolombia.com/medicina/ neumologia/neumologia16304-tuberculosis.htm

22. Abughali N, van der Kuyp F, Annable W, Kumar ML. Congenital tuberculosis. Pediatr Infect Dis J. 1994;13:738-41.

23. Ray M, Dixit A, Taipei K, Singhi PD. Congenital tuberculosis. Indian Pediatrics. 2002;39:1167-8.

24. Mazade MA, Evans EM, Starke JR, Correa AG. Congenital tuberculosis presenting as sepsis syndrome: case report and review of the literature. Pediatr Infect Dis J. 2001;20:439-42.

25. Chen A, Shih S. Congenital tuberculosis in two infants. AJR Am J Roentgenol. 2004;182:253-6.

26. Cegielski JP, Chin DP, Espinal MA, Frieden TR, Rodríguez-Cruz R, Talbot EA, et al. The global tuberculosis situation. Progress and problems in the 20th century, prospects for the 21st century. Infect Dis Clin North Am. 2002;16:1-58.

27. Pillet $P$, Grill J, Rakotonirina G, Holvoet-Vermaut L, Auregan G, Guyon P. Congenital tuberculosis: difficulties in early diagnosis. Arch Pediatr. 1999;6:635-9.

28. Cunningham DG, McGraw TT, Griffin AJ, O’Keefe JP. Neonatal tuberculosis with pulmonary cavitation. Tubercle. 1987;63:217-22.

29. Amick FE, Alden MW, Sweet LK. Congenital tuberculosis. Pediatrics. 1950;6:384-90.

30. lademarco MF, Castro KG. Epidemiology of tuberculosis. Semin Respir Infect. 2003;18:225-40.

31. Council of the Infectious Disease Society of America. Diagnostic standards and classification of tuberculosis in adults and children. Am J Respir Crit Care Med. 2000;161:1376-95. 